\title{
GW23-e1413 HIGH PREVALENCE OF TYPE-2 DIABETES IN CHINESE OIL WORKERS: INTERACTION BETWEEN SAA1 GENE AND WORK STRESS
}

\author{
doi:10.1136/heartjnl-2012-302920a.92
}

Xiang Xie, Yi-Tong Ma, Yi-Ning Yang, Xiao-Mei Li, Yi-Tong Ma. Department of Cardiology, First Affiliated Hospital of Xinjiang Medical University, Urumqi, 830054 P.R. China

Objectives Serum Amyloid A (SAA) was reported associated with insulin resistance and type- 2 diabetes. The present study aimed to investigate the prevalence of type- 2 diabetes and its association with SAA1 genetic polymorphisms in Chinese oil workers.

Methods Three stages were performed for the present study. In the stage one, a cross-sectional survey was designed to investigate the prevalence of type- 2 diabetes in oil workers; in the stage two, we detected the SAA1 genetic polymorphisms and analysed their association with serum glucose (GLU) levels; in the stage three, we designed a nested case-control study to analyse the association of diabetes with SAA1 gene polymorphisms.

Results Overall, the prevalence of type- 2 diabetes was $15.6 \%$ in total, $14.9 \%$ in men, and $18.0 \%$ in women, respectively. In nondiabetic individuals, rs2229338, rs4638289 and rs12218 were found to be significantly associated with serum GLU levels before and after multivariate adjustment (all $\mathrm{P}<0.05$ ). In the nested case-control study, we found rs2229338, rs12218, and rs11603089 was associated with Type-2 diabetes by univariate analysis, respectively (all $p<0.05$ ). After adjustment of confounders, the difference remained significant in rs2229338 $(\mathrm{p}=0.015, \quad \mathrm{OR}=2.610 \quad(95 \% \mathrm{CI}: 1.204$ to 5.656$))$ and $\mathrm{rs} 12218$ $(\mathrm{P}=0.018, \mathrm{OR}=2.797$ (95\% CI: 1.197 to 6.537)). Furthermore, there was a significant interaction between rs2229338 and work stress on type 2 diabetes ( $p=0.001, O R=2.304$ (95\% CI: 1.387 to 3.829$)$ ).

Conclusions Type 2 diabetes is highly prevalent in Chinese oil workers. The genetic polymorphisms of SAA1 were associated with serum glucose levels in nondiabetics and were independent risk factors of type 2 diabetes in Chinese oil workers. 